\title{
EFFICACY OF PRAZIQUANTEL LOADED CHITOSAN NANOPARTICLES AGAINST BOTH ADULT AND SCHISTOSOMULA STAGES OF SCHISTOSOMA MANSONI
}

\section{By}

GEHAN SALAH SADEK, NANCY MAHMOUD HARBA, SAMAR AHMED ELREFAI, SHAIMAA AHMED SHARAF EL-DEEN ${ }^{*}$ and MONA MOHAMED SALEH Department of Medical Parasitology, Faculty of Medicine, Menoufia University, Shebin el-kom, Menoufia, Egypt

(Correspondence: drsharaf81@yahoo.com, 00201001118501, samarelrefai5@gmail.com)

\section{Abstract}

Schistosomiasis is the $2^{\text {nd }}$ most common parasitic disease that causes around 300000 deaths annually worldwide. The main antischistosomal therapy; praziquantel (PZQ) has many imperfections e.g. resistance, frail impact against premature stages and many side effects. This raised the need to find a substitute or to repair PZQ defects. This work aimed to assess value of loading PZQ to chitosan nanoparticles (CS-NPs) regarding usage of lower PZQ dose (to decrease side effects) and broadening its spectrum (to involve premature stages of Schistosoma mansoni).

This study involved 11 mice groups. The first four groups served for schistosomula studies (mice were euthanized 17 days post infection (d.p.i.). Group I served as positive schistosomula controls. Groups II, III and IV received pre-maturation treatments 10 d.p.i. The last seven groups served for adult worm studies (mice were euthanized 50 d.p.i.). Group V served as positive adult controls. Groups VI, VII and VIII received pre-maturation treatments 10 d.p.i. Groups IX, X and XI received post-maturation treatments 45 d.p.i.

Drug efficacy against schistosomula was assessed by counting and scanning electron microscopy (SEM) of the retrieved schistosomula. Efficacy against adult worms was assessed by studying worm load, ova count, oogram, granuloma studies and SEM of adult worms. Results showed that, PZQ loaded CS-NPs (PZQ/CS-NPs) significantly reduced schistosomula count with no morphological changes. Regarding adults, PZQ/CS-NPs achieved a statistically significant higher reduction of all assessed parameters - compared to traditional PZQ - especially when administered as a post-maturation treatment. Regardless of absent morphological changes of schistosomula, clear morphological deformities appeared when these schistosomula were permitted to develop into adults.

Key Words: Praziquantel; Chitosan; Nanoparticles; Schistosoma; schistosomulum.

\section{Introduction}

Schistosomiasis is the second most common human parasitic disease worldwide. Reports of World Health Organization (WHO) state that more than 240 million individuals are infected with Schistosoma $(S$.) species and over 700 millions are at risk of infection in endemic areas. Its mortality records have surpassed 200000 individuals in sub Saharan Africa alone with global annual mortality ranging from 280000 to 500000 (WHO, 2010).

Humans can be infected by three main species, S. haematobium, S. mansoni or S. japonicum (WHO, 2016). Infection begins by penetration of skin and/or mucous membranes with Schistosoma cercariae upon contact with contaminated water. Once penetration occurs, cercariae lose their tails and find an access to the circulation to lodge in lung vessels as pulmonary schistosomula ( $\mathrm{He}$ et al, 2002; 2005). After a period of around 15 days, schistosomula reach their final habitat which is either urogenital venous plexus -in $S$. haematobium- or mesenteric venous plexus -in other species-. Another 10 days usually pass before the juveniles mature into adults capable of pairing and laying ova (Gobert et al, 2007) that may lodge in tissues causing granulomas and fibrosis of 
many vital organs (Pearce and MacDonald, 2002; Burke et al, 2009).

Many remedies were used to get rid of this pathogen but praziquantel (PZQ) is still the mainly used official therapy. Unfortunately, it doesn't perfectly kill the premature stages of Schistosoma and acts only after their maturation and start of laying ova which by itself is not completely killed by PZQ (de Oliveira et al, 2014). This occurs because PZQ is immediately transformed into inactive or less effective form after its administration (Tomiotto-Pellissier et al, 2017). The low water solubility of PZQ markedly influences its absorption with subsequent need for higher doses - and eventually extra hazards - to get a perfect action (Omar et al, 2005; Passerini et al, 2006). In addition to tolerance and resistance that were reported in some cases (da Silva et al, 2017).

Absence of an intense medication that can supplant PZQ defects encouraged studies to improve its pharmacokinetics. Nanoparticles (NPs) have the potential to deliver toxic components or drugs to the target pathogen without harming the host tissues by improving pharmacokinetics of drugs (Bergquist $e t$ $a l$, 2017). Among the various sorts of NPs, polymeric NPs are considered a critical type because of their good biocompatibility, biodegradability and available formulation methods. Chitosan (CS) based polymeric NPs have been recently introduced to the field of nanotherapeutics as an important candidate for drug delivery (Mohammed et al, 2017).

Chitosan is produced from chitin of crustacean shell and fungal cell walls (Berger et al, 2004; Tikhonov et al, 2006). It proved many antimicrobial, immune stimulant and cancer resisting properties. Its low toxicity and muco-adhesive properties made CS itself and its NPs good candidates for drug delivery systems. They act as penetration enhancers by opening the tight epithelial junctions (Pattani et al, 2009; Abdel-Latif et al, 2017; Mohammed et al, 2017; Umair et $a l$, 2017). They proved better stability and cellular uptake than solid lipid nanoparticles
(SLNs) (Wang et al, 2014; Luo et al, 2015).

The present work was designed to assess the value of loading PZQ to CS-NPs, regarding the use of a lower dose to decrease risk of its adverse effects and broadening its spectrum to involve premature schistosomula stages, thus mending two major defects in the main antischistosomal drug, PZQ.

\section{Materials and Methods}

Ethics statement: All animal experiments were performed at Theodor Bilharz Research Institute, TBRI (Giza, Egypt). Mice were kept under standard housing conditions and standard commercial diet in animal house of TBRI. Room temperature was kept at 20$22^{\circ} \mathrm{C}$. Experimental procedures were performed in accordance with international ethical guidelines after approval of institutional ethical committee of TBRI. Also, the authors assert that all procedures contributing to this work comply with the ethical standards of relevant national and institutional guides on the care and use of laboratory animals.

Study design: The mice groups were used to study drug efficacy on two developmental stages of $S$. mansoni; schistosomula and adults. That's why two types of studies were performed. Schistosomula included the first four groups and their mice were euthanized 17 d.p.i., and Adult worms: included the last seven groups that received either pre- or post-maturation treatment, and their mice were euthanized 50 d.p.i.

Schistosomula (mice euthanized 17 d.p.i., of 10mice each): GI: S. mansoni infected un treated mice (positive schistosomula controls). GII: $S$. mansoni infected then received PZQ full dose at $10^{\text {th }}$ day p.i. $\left(\mathrm{PZQ}^{10 / 17}\right)$, GIII: $S$. mansoni infected then received PZQ /CS-NPs at day 10 p.i. (PZQ/Cs-NPs ${ }^{10 / 17}$ ), GIV: S. mansoni infected then received unloaded CS-NPs at day 10 p.i. $\left(\mathrm{CS}^{10 / 17}\right)$.

Adults (mice euthanized 50 d.p.i. of $10 \mathrm{mi}$ ce each): GV: S. mansoni infected untreated mice (Positive adult controls), GVI: S. mansoni infected then received a pre-maturation treatment of PZQ at day 10 p.i. $\left(\mathrm{PZQ}^{10 / 50}\right)$, GVII: S. mansoni infected then received a 
pre-maturation treatment of $\mathrm{PZQ} / \mathrm{CS}-\mathrm{NPs}$ at day 10 p.i. (PZQ/CS-NPs ${ }^{10 / 50}$ ), GVIII: $S$. mansoni infected then received pre-maturation treatment of unloaded CS-NPs at day 10 p.i. $\left(\mathrm{CS}^{10 / 50}\right)$, GIX: S. mansoni infected then received a post-maturation treatment of PZQ full dose at day 45 p.i. (Traditional PZQ treatment; PZQ ${ }^{45 / 50}$ ), GX: $S$. $m a$ nsoni infected then received a post-maturation treatment of PZQ/CS-NPs at day 45 p.i. (PZQ/CS-NPs ${ }^{45 / 50}$ ), GXI: S. mansoni infected then received a post-maturation treatment of unloaded CS-NPs at day 45 p.i. $\left(\mathrm{CS}^{45 / 50}\right)$.

Experimental animals and S. mansoni infection: 110 male pathogen free BALB/c mice (6-8 weeks old, 18-20 gm) were infected by subcutaneous injection of $80-100$ cercariae/mouse of an Egyptian strain of $S$. mansoni (Peters and Warren, 1969).

Praziquantel treatment: PZQ (Egyptian International Pharmaceutical Company, E.I.P. C.O.) was suspended in $2 \%$ cremophore (Sigma Aldrich, USA). It was administered orally starting at day 10 p.i. for 2 consecutive days at a dose of $500 \mathrm{mg} / \mathrm{kg}$ (El-Lakkany et al., 2012) by GII and GVI and 45 d.p.i. by GIX (Pearce and James, 1986).

Chitosan nanoparticles preparation and loading with PZQ: CS-NPs were purchased from TBRI Parasitology Department. CSNPs were synthesized via the ionotropic gelation of CS with tripolyphosphate (TPP) anions (Gaafar et al, 2014).

PZQ/CS-NPs were formed by addition of CS solution to TPP solution containing 250 mg concentrations of PZQ. PZQ/CS-NPs were separated from the aqueous suspension by centrifugation at $13,000 \mathrm{~g}$ and $4^{\circ} \mathrm{C}$ for 30 minutes. The supernatant was collected, its protein content (i.e. PZQ) was determined by Bradford protein assay spectrophotometric method at $595 \mathrm{~nm}$ (Ding et al, 2017).

Unloaded CS-NPs were orally administered started at day 10 p.i. for 2 consecutive days (El-Lakkany et al., 2012) by GIV, GVIII and 45 d.p.i. (Pearce and James, 1986) by GXI at a dose of $33 \mathrm{mg} / \mathrm{kg}$ (Akhtar et al, 2012). PZQ/CS-NPs were orally administrated started at day 10 p.i. for 2 consecutive days by GIII and GVII and 45 d.p.i. by GX at a dose of $250 \mathrm{mg} / \mathrm{kg}$ (half of the traditional dose).

Euthanizing mice and sample collection: At day 17 p.i., mice groups of schistosomula study (from GI to GIV) were decapitated then post lung schistosomula were retrieved by liver perfusion (Pearce and James, 1986). Retrieved schistosomula were counted then preserved in glutaraldehyde for further electron microscopic studies.

At day 50 p.i., mice groups of adult worm studies (GV to GXI) were decapitated. Adult worms were retrieved by liver perfusion as above. Intestinal (ileum) and liver and samples were removed for tissue egg load and oogram studies. Liver samples were removed, rinsed with physiological saline and preserved in formalin $10 \%$ for histopathological studies. Retrieved adult worms were preserved in glutaraldehyde for further electron microscopic studies.

Schistosomula count: For groups I, II, III \& IV, retrieved schistosomula were collected in petri dishes and counted using a stereoscopic microscope.

SEM of schistosomula: Retrieved schistosomula were fixed with $2.5 \%$ (v/v) glutaraldehyde in a phosphate-buffered saline (PBS, $\mathrm{pH}$ 7.4) for $24 \mathrm{~h}$ at room temperature. Later, they were rinsed three times with PBS before storage in PBS at $4^{\circ} \mathrm{C}$ until use. Before SEM, schistosomula were twice washed with distilled water, dehydrated in ascending ethanol concentrations, and critically point dried. They were placed on aluminum stubs, sputter coated with $20 \mathrm{~nm}$ gold nanoparticles, and examined in a high-resolution SEM (jol $5200 \mathrm{lv}$, Japan) at an accelerating voltage of $5 \mathrm{kV}$ in Electron Microscope Unit, Faculty of Medicine, Tanta University (Manneck et al, 2011).

Adult worms count: Adults S. mansoni were recovered from livers of groups $\mathrm{V}$ to $\mathrm{XI}$ by normal saline perfusion of hepatic and porto-mesenteric vessels through cannula- 
tion of inferior vena cava of euthanized mice. Then, male, female and coupled worms were counted using a stereoscopic microscope (Duvall and DeWitt, 1967).

Tissue egg load and oogram studies: Liver and intestinal (ileum) samples of GV to GXI were collected, weighed and digested in 5\% $\mathrm{KOH}$ at $37^{\circ} \mathrm{C}$ for 16 hours. Ova were counted using light microscopy at $\mathrm{x} 40$. Developmental stages of ova were identified for oogram studies (Pellegrino et al, 1962; Herbert et al, 2010).

Hematoxylin and eosin (H\&E) staining of liver for granuloma studies: Liver samples of euthanized mice (GV to GXI) were fixed in formalin $10 \%$, paraffinized and stained with H\&E (Goyal et al, 2013). Granulomas were counted, and their diameters were digitally measured using a multi-head microscope, Olympus SC100 and analysis getIT software. Diameter of granuloma with a single ovum was the only considered.

SEM adult studies: Isolated adult worms (GV to GXI) were prepared and examined by SEM as mentioned above for schistosomula.

Statistical analysis: Data entry, coding, and analysis were conducted using SPSS (20), IBM Corp. Released 2011. IBM SPSS Statistics for Windows, Version 20.0. Armonk, NY: IBM Corp. Data of this study were of both quantitative and qualitative types. Quantitative data were expressed in mean $(\overline{\mathrm{x}})$, and standard deviation (SD). ANOVA (for parametric data) and Kruskal Wallis (for non-parametric data) tests were used to estimate the difference between means of more than two quantitative variables. Qualitative data were expressed as number and percent (\%). Chi-square test $\left(X^{2}\right)$ was used to assess the relationship between qualitative parameters. The level of significance of our data was $95 \%$, so, $p$ value $>0.05$ was considered a non-statistically significant difference, while $\mathrm{p}$ value $<0.05$ was considered significant difference.

\section{Results}

Pre-maturation euthanasia of mice (GI to
GIV, 17 d.p.i.) showed that, schistosomula count was significantly reduced in both groups that received CS-NPs either sole or loaded with PZQ compared to PZQ treated group $(p<0.01)$ without significant difference between the former two groups (Fig. 1). No morphological changes were detected by SEM in any of the schistosomula of all treated groups.

Administration of PZQ/CS-NPs as a postmaturation treatment achieved the highest reduction percent of total worm load with statistically significant differences when compared to other treated groups $(\mathrm{p}<0.01)$ followed by pre-maturation PZQ/CS-NPs which showed no significant difference when compared to traditional PZQ ( $p>0.05)$. Similar results were recorded regarding male, female and coupled worm loads (Fig. 2).

Reduction of PZQ/CS-NPs to worm load was reflected on numbers of the laid ova. So, similar to worm load, post-maturation treatment with PZQ/CS-NPs achieved the highest percent of both intestinal and liver egg load reduction followed by prematuration $\mathrm{PZQ} / \mathrm{CS}-\mathrm{NPs}$ treatment with a statistically significant difference between both groups $(p<0.001)$. Despite that difference, both groups were statistically higher when compared to other studied groups including traditional PZQ treated group that ranked $3^{\text {rd }}(\mathrm{p}<0.01)$ (Fig. 3).

The killing efficacy of PZQ/CS-NPs exceeded adult worms to involve the laid eggs. So, the highest percent of dead eggs was recorded with post-maturation treatment with PZQ/CS-NPs followed by traditional PZQ with a statistically significant difference between both groups $(p<0.001)$. Prematuration PZQ/CS-NPs treated group ranked $3^{\text {rd }}$ with a significant difference compared to the former two groups $(p<0.001)$. The reverse was detected regarding percent of mature eggs (Fig. 4).

The reduction of tissue egg load by postmaturation PZQ/CS-NPs was presented histopathologically in the form of reduced 
number of hepatic granulomas that was subsequently the highest reduction followed by traditional PZQ treatment when compared to other treated groups $(\mathrm{p}<0.01)$ with a statistically significant difference between both groups $(\mathrm{p}<0.001)$. Mice which received prematuration treatment with PZQ/CS-NPs ranked $4^{\text {th }}$ after sole post-maturation CS-NPs treated group with a statistically significant difference between both groups $(\mathrm{p}<0.001)$.

Effects of post-maturation treatment with PZQ/CS-NPs on liver granulomas exceeded reduction of their numbers to involve their diameters and possessed the $1^{\text {st }}$ rank with a statistically significant difference when compared to all other studied groups. The second rank was for both sole CS-NPs postmaturation treatment and traditional PZQ with no statistically significant difference between both groups ( $p>0.05$ ) (Figs. 5, 6).

As regards SEM, adults revealed marked deformities of tegument and suckers in the form of loss of normal tegument architecture, rough tegument, erosion of some tubercles, loss of spines, and swelling of tubercles. Sucker changes appeared as blebs formation, distortion of ventral sucker shape and disturbance in direction of spines. These changes were observed in groups that received either pre- or post-maturation treatments either with PZQ/CS-NPs or sole CSNPs. These deformities were less severe in the group that received traditional PZQ and totally absent in the group that received prematuration PZQ treatment. Gynecophoric canal changes were detected with all postmaturation treatments especially $\mathrm{PZQ} / \mathrm{CS}$ NPs in the form of roughness of canal with blebs formation. Swelling deformity of the whole worm was only detected in prematuration CS-NPs treated groups either sole or loaded with PZQ. It was detected in both groups (Fig. 7).

\section{Discussion}

In the present study, schistosomula count was significantly reduced in groups that received CS-NPs either sole or loaded with PZQ as compared to positive controls and
PZQ treated ones. PZQ treatment did not cause any significant reduction of schistosomula count when compared to positive control group. This agreed with Vimieiro $e t$ al. (2013) who found that PZQ administered at the day 14 p.i. caused only little reduction of immature $S$. mansoni worms. Contrarily, Issa (2007) reported that pre-maturation traditional PZQ treated gave significant reduction of schistosomula count as compared to positive controls.

In the present study, there were no detectable SEM changes in schistosomula of all treated groups. Morphological changes were only revealed after reaching adult stage in groups that received pre-maturation CS-NPs treatment either alone or loaded with PZQ when mice were euthanized 50 d.p.i. Such adult changes were in the form of edema and whole tegument swelling. But, Silva et al. (2003) recorded relative resistance of the larval stages of $S$. mansoni to schistosomicidal drugs. On contrary, immunofluorescence study done by Flisser and McLaren (1989) after recovering of lung-stage larvae of $S$. mansoni after treatment with PZQ, revealed mild tegumental lesions with exposure of parasite antigens.

In the present study, despite using PZQ only in half dose, PZQ/CS-NPs achieved the highest reduction percent of both worm and egg loads whether given as a pre- or postmaturation treatment preceding traditional PZQ that ranked $3^{\text {rd }}$ in most of comparisons.

To the authors' knowledge, none usage of CS-NPs as delivery vehicles for PZQ but many studies used it for other drugs that agreed with the ability of CS-NPs increased therapeutic effect of the drugs loaded. Du et al. (2009) reported improved antibacterial properties of many metal ions when loaded to CS-NPs especially against gram negative bacteria. Also, Akhtar et al. (2012) reported a significant improvement of curcumin loaded CS-NPs' bioavailability in addition to decrease degradation and better uptake by RBCs compared to free curcumin in malaria treatment. Ability of CS-NPs to enhance cel- 
lular internalization of drugs was reported by Chaubey and Mishra (2014) on Leishmania. They found that Mannose conjugated CS-NPs assisted delivery of rifampicin to the interior of macrophages which acted as a shield for the parasite against the host's immune system. Jain et al. (2014) reported better internalization of the anti-leishmanial drug, amphotericin B into murine macrophage cell culture when the carriers SLNs were coated with $\mathrm{CS}$.

de Souza et al. (2014) reported improved schistosomicidal activity of PZQ loaded on SLNs at lower concentrations than free PZQ, and improved intestinal permeation and lowered cytotoxicity on cultured hepatocytes than free PZQ. It worth mentioning that Ying et al. (2011); Wang et al. (2014) and Luo et al. (2015), reported that CS coating improved stability, drug loading, release and cellular uptake of SLNs themselves.

Frezza et al. (2013) studied the effect of encapsulating PZQ within liposome NPs and reported highest percent of worm load reduction achieved by full dose liposome NP encapsulated PZQ either given before or after adults' maturation. The same was recorded for ova count. Compared to our results, CS-NPs worked well with only half dose of PZQ. This can raise an idea that the synergistic effects of CS-NPs are better than liposome-based NPs.

In the present work, the mean number and size of hepatic granulomas were the least in mice group that received post- maturation PZQ/CS-NPs. Granuloma number reduction was explained by decreased ova count caused by this drug while decreased granuloma size was due to the CS anti-inflammatory properties (Davydova et al, 2016).

In the present study, SEM of adults revealed severe tegument and sucker changes with pre- and post-maturation treatment with PZQ/CS-NPs in addition to pre- and postmaturation treatment with sole CS-NPs that also induced similar changes. These findings explained the reduction of worm load and ova count because tegument deformities lead to increased exposure of Schistosoma antigens to be readily destroyed by host immune response especially with immune stimulating agent, CS (Smith et al, 2014). Frezza et al. (2015) found severe tegument and sucker changes in $S$. mansoni infected mice treated with liposome encapsulated PZQ in presence of hyperbaric oxygen.

\section{Conclusion}

Loading PZQ to CS-NPs improves PZQ effects on the premature stage; schistosomulum. Also, it improved all adult therapeutic properties of PZQ even when used in half the therapeutic dose. Its effects on morphology are detected after worm maturation in the form of swelling deformities of adults besides tegument, sucker and gynecophoric canal changes.

Conflict of Interest: The authors declared that they neither have competing interests nor received fund.

\section{Acknowledgement}

The authors would like to thank Dr. Taysseer Abd EL Hamid Ibrahim, Assistant Lecturer of Pathology, Menoufia Liver Institute for her kind help in interpretation of histopathology data.

\section{References}

Abdel-Latif, M, El-Shahawi, G, Aboelhadid, SM, Abdel-Tawab, H, 2017: Immunoprotective effect of chitosan particles on hymenolepis nanainfected mice. Scand. J. Immunol. 86, 2:83-90.

Akhtar, F, Rizvi, MM, Kar, SK, 2012: Oral delivery of curcumin bound to chitosan nanoparticles cured Plasmodium yoelii infected mice. Biotechnol. Adv. 30, 1:310-20.

Berger, J, Reist, M, Mayer, JM, Felt, O, Peppas, NA, et al, 2004: Structure and interactions in covalently and ionically crosslinked chitosan hydrogels for biomedical applications. Euro J. Pharmaceut. Biopharmaceut. 57, 1:19-34.

Bergquist, R, Utzinger, J, Keiser, J, 2017: Controlling schistosomiasis with praziquantel: How much longer without a viable alternative? Infect.Dis. Poverty 6, 1:74.

Burke, ML, Jones, MK, Gobert, GN, Li, YS, Ellis, MK, et al, 2009: Immunopathogenesis of human schistosomiasis. Parasit. Immunol. 31: 163-76.

Chaubey, P, Mishra, B, 2014: Mannose-conju- 
gated chitosan nanoparticles loaded with rifampicin for the treatment of visceral leishmaniasis. Carbohydrate Polymers 101:1101-8.

da Silva, VBR, Campos, BRKL, de Oliveira, J F, Decout, JL, do Carmo, et al, 2017: Medicinal chemistry of antischistosomal drugs: Praziquantel and oxamniquine. Bioorgan. Med. Chem. 25, 13:3259-77.

Davydova, VN, Kalitnik, AA, Markov, PA, Volod'ko, AV, Popov, SV, et al, 2016: Cytokine-inducing and anti-inflammatory activity of chitosan and its low-molecular derivative. Appl. Biochem. Microbiol. 22, 5:476-82.

de Oliveira, RN, Rehder, VL, Oliveira, AS, Jeraldo, L, Linhares, AX, et al, 2014: Anthelmintic activity in vitro and in vivo of Baccharis trimera (Less) DC against immature and adult worms of Schistosoma mansoni. Exp. Parasitol. 139:63-72

de Souza, AL, Andreani, T, de Oliveira, RN, Kiill, CP, dos Santos, FK, et al, 2014: In vitro evaluation of permeation, toxicity and effect of praziquantel-loaded solid lipid nanoparticles against Schistosoma mansoni as a strategy to improve efficacy of the schistosomiasis treatment. Inter. J. Pharmaceut. 463, 1:31-7.

Ding, RL, Xie, F, Hu, Y, Fu, SZ, Wu, JB, et al, 2017: Preparation of endostatin-loaded chitosan nanoparticles and evaluation of the antitumor effect of such nanoparticles on the Lewis lung cancer model. Drug Delivery 24, 1:300-8.

Du, W, Niu, S, Ying-Lei, Y, Xu, Z, Fan, C, 2009: Antibacterial activity of chitosan tripolyphosphate nanoparticles loaded with various metal ions. Carbohydrate Polymers 75:385-9.

Duvall, RH, DeWitt, WB, 1967: An improved perfusion technique for recovering adult Schistosomes from laboratory animals. Am. J. Trop. Med. Hyg. 16:483-6.

El-Lakkany, NM, Hammam, OA, El-Maadawy, WH, Badawy, AA, Ain-Shoka, AA, et al, 2012: Anti-inflammatory/anti-fibrotic effects of the hepatoprotective silymarin and the schistosomicide praziquantel against Schistosoma manso$n i$-induced liver fibrosis. Parasit. Vectors 5:9-11. Flisser, A, McLaren, DJ, 1989: Effect of praziquantel treatment on lung stage larvae of Schistosoma mansoni in vivo. Parasitol. 98: 203-11.

Frezza, TF, de Souza, AL, Prado, CC, de Oliveira, C, Gremião, MP, et al, 2015: Effectiveness of hyperbaric oxygen for experimental treatment of Schistosoma mansoni using praziquantel-free and encapsulated into liposomes: Assay in adult worms and oviposition. Acta Trop. 150: 182-9.

Frezza, TF, Gremião, MP, Zanotti-Magalhã es, EM, Magalhães, LA, de Souza, AL, Allegretti, SM, 2013: Liposomal-praziquantel: efficacy against Schistosoma mansoni in a preclinical assay. Acta Trop. 128, 1:70-75.

Gaafar, MR, Mady, RF, Diab, RG, Shalaby, TI, 2014: Chitosan and silver nanoparticles: promising anti-Toxoplasma agents. Exp. Parasitol. 143:30-8.

Gobert, GN, Chai, M, McManus, DP, 2007: Biology of the schistosome lung-stage schistosomulum. Parasitol. 134:453-60.

Goyal, N, Rishi, P, Shukla, G, 2013: Lactobacillus rhamnosus GG antagonizes Giardia intestinalis induced oxidative stress and intestinal disaccharidases: an experimental study. Wld. J. Microbiol. Biotechnol. 29, 6:1049-57.

He, YX, Chen, L, Ramaswamy, K, 2002: Schistosoma mansoni, S. haematobium, and S. japonicum: early events associated with penetration and migration of schistosomula through human skin. Exp. Parasitol. 102:99-108.

He, YX, Salafsky, B, Ramaswamy, K, 2005: Comparison of skin invasion among three major species of Schistosoma. Trends Parasitol. 21: 201-3.

Herbert, DR, Orekov, T, Roloson, A, Ilies, M, Perkins, C, et al, 2010: Arginase I suppresses IL-12/IL-23p40- driven intestinal inflammation during acute schistosomiasis. Immunol. J. 184, 11:6438-46.

Issa, RM, 2007: Schistosoma mansoni: Prophylactic and curative effect of propolis in experimentally mice. Rawal Medical J. 32, 2:94-8.

Jain, V, Gupta, A, Pawar, VK, Asthana, S, Jaiswal, AK, et al, 2014: Chitosan-assisted immunotherapy for intervention of experimental leishmaniasis via amphotericin B-loaded solid lipid nanoparticles. Appl. Biochem. Biotechnol. 174, 4:1309-30.

Luo, Y, Teng, Z, Li, Y, Wang, Q, 2015: Solid lipid nanoparticles for oral drug delivery: Chitosan coating improves stability, controlled delivery, mucoadhesion and cellular uptake. Carbohydrate Polymers 122:221-9.

Manneck, T, Braissant, O, Ellis, W, Keiser, J 2011: Schistosoma mansoni: Antischistosomal activity of the four optical isomers and the two racemates of mefloquine on schistosomula and adult worms in vitro and in vivo. Exp. Parasitol. 127, 1:260-9. 
Mohammed, MA, Syeda, JTM, Wasan, KM, Wasan, EK, 2017: An overview of chitosan nanoparticles and its application in non-parenteral drug delivery. Pharmaceut. 9, 4:E53.

Omar, A, Elmesallamy, GS, Eassa, S 2005: Comparative study of the hepatotoxic, genotoxic and carcinogenic effects of praziquantel distocide $\&$ the natural myrrh extract Mirazid on adult male albino rats. J. Egypt. Soc. Parasitol. 35: 313-29.

Passerini, N, Albertini, B, Perissutti, B, Rodriguez, $\mathbf{L}, 2006$ : Evaluation of melt granulation and ultrasonic spray congealing as techniques to enhance the dissolution of praziquantel. Inter. J. Pharmaceut. 318:92-102.

Pattani, A, Patravale, VB, Panicker, L, Potdar, PD, 2009: Immunological effects and membrane interactions of chitosan nanoparticles.

Mol. Pharmaceut. 6, 2:345-52.

Pearce, EJ, James, SL, 1986: Post lung-stage schistosomula of Schistosoma mansoni exhibit transient susceptibility to macrophage-mediated cytotoxicity in vitro that may relate to late phase killing in vivo. Parasite Immunol. 8, 5:513-27.

Pearce, EJ, MacDonald, AS, 2002: The immunobiology of schistosomiasis. Nat. Rev. Immunol. 2:499-511.

Pellegrino, J, Oliveira, CA, Faria, J, Cunha, AS, 1962: New approach to the screening of drugs in experimental schistosomiasis mansoni in mice. Am. J. Trop. Med. Hyg. 11:201-15.

Peters, PA, Warren, KS, 1969: A rapid method of infecting mice and other laboratory animals with Schistosoma mansoni: subcutaneous injection. Parasitol. 55, 3:55-8.

Silva, LM, Menezes, RM, Oliveira, SA, Andrade, ZA 2003: Chemotherapeutic effects on larval stages of Schistosoma mansoni during infection and re-infection of mice. Rev. Soc. Brasil. Med. Trop. 36, 3:335-41.

Smith, A, Perelman, M, Hinchcliffe, M 2014:
Chitosan: A promising safe and immune-enhancing adjuvant for intranasal vaccines. Hum. Vac. Immunotherapeut. 10, 3:797-807.

Tikhonov, VE, Stepnova, EA, Babak, VG, Yamskov, IA, Palma-Guerrero, J, et al, 2006: Bactericidal and antifungal activities of a low molecular weight chitosan and its $\mathrm{N}-/ 2$ (3)-(dodec-2-enyl) succinoyl/-derivatives. Carbohydrate Polymers 64:66-72.

Tomiotto-Pellissier, F, Miranda-Sapla, MM, Machado, LF, Bortoleti, BTDS, Sahd, CS, et al, 2017: Nanotechnology as a potential therapeutic Alternative for schistosomiasis. Acta Trop. 174:64-71.

Umair, S, Pernthaner, A, Deng, Q, Gibson, B, Hook, S Heath, D, 2017: Preliminary evaluation of a thermosensitive chitosan hydrogel for Echinococcus granulosus vaccine delivery. Vet. Parasitol. 236:117-20.

Vimieiro, AC, Araújo, N, Katz, N, Kusel, J, Coelho, PM, 2013: Schistogram changes after administration of antischistosomal drugs in mice at the early phase of Schistosoma mansoni infection. Mem. Inst. Oswaldo Cruz. 108, 7:881-6.

Wang, F, Chen, L, Zhang, D, Jiang, S, Shi, K, et al, 2014: Methazolamide-loaded solid lipid nanoparticles modified with low-molecular weight chitosan for the treatment of glaucoma: vitro and vivo study. J.Drug Target. 22, 9:849-58.

WHO, 2010: Schistosomiasis. Epidemiological situation.http://www.who.int/schistosomiasis/epidemiology/en/

WHO, 2016: Neglected tropical diseases. Egypt leverages domestic funding to eliminate schistosomisis.http://www.who.int/neglected diseases/ news/Egypt_leverages_domestic_funding_to_eli minate schistosomiasis/en/

Ying, XY, Cui, D, Yu, L, Du, YZ, 2011: Solid lipid nanoparticles modified with chitosan oligosaccharides for the controlled release of doxorubicin. Carbohydrate Polymers 84, 4:1357-64

\section{Explanation of figures}

Fig. 1: Comparison between groups of schistosomula regarding mean schistosomula count. N.B. Groups with same letter without significant difference, those with different letters with significant difference. $\mathrm{PR}=$ reduction $\%$.

Fig. 2: Comparison between mean adult worm loads in groups of adult worms. N.B. Groups with same letter without significant difference, those with different letters with significant difference. $\mathrm{PR}=$ reduction \%.

Fig. 3: Comparison between mean ova count in intestinal and hepatic tissues in adult worms. N.B. Groups with same letter without significant difference, those with different letters with significant difference. $\mathrm{PR}=$ reduction $\%$.

Fig. 4: Comparison between oogram patterns in adult worms. N.B. Groups with same letter without significant difference while those with different letters with significant difference. $\mathrm{PR}=$ reduction \%.

Fig. 5: Comparison between mean hepatic granulomas number and diameter in adult worms. N.B. Groups with same letter without significant difference while those with different letters with significant difference. $\mathrm{PR}=$ reduction \%.

Fig. 6: a- Liver tissue of positive adult control showed large sized fibrocellular granuloma surrounding S. mansoni ova (Green arrow), b-

Liver tissue of a mouse treated with traditional PZQ showed moderate sized granuloma surrounding ova. c- Liver tissue of a mouse treated with post- maturation PZQ/CS-NPs showed small sized granuloma surrounding ova $(\mathrm{H} \& \mathrm{E}$ stain; scale bar $=100 \mu \mathrm{m})$. 
Fig.7: a- SEM of normal tegument of male $S$. mansoni from positive adult control. Regularly arranged coarse tubercles bearing spines with naked apex, Spines sharp and apically directed (x1000), b- SEM of tegument of male S. mansoni received post- maturation PZQ/CS-NPs showed spines loss (x1000), c- SEM of $S$. mansoni from positive adult control showed normal oral sucker concerning shape, thickness and direction of spines (inwards toward mouth) and normal ventral sucker (tyre like with numerous hairy spines on internum \& outer prominent rim) (x350), d- SEM of $S$. mansoni suckers from group received post-maturation unloaded CS-NPs showed spines loss of oral and ventral suckers (x700), e- SEM S. mansoni positive control showed normal gynecophoric canal (x1000), f- SEM of S. mansoni received postmaturation PZQ/CS-NPs showed roughness of gynecophoric canal (x1000), g- SEM of male $S$. mansoni positive adult control showed normal size (x100), h-SEM of male $S$. mansoni received pre-maturation PZQ/CS-NPs showed marked swelling (x1000).
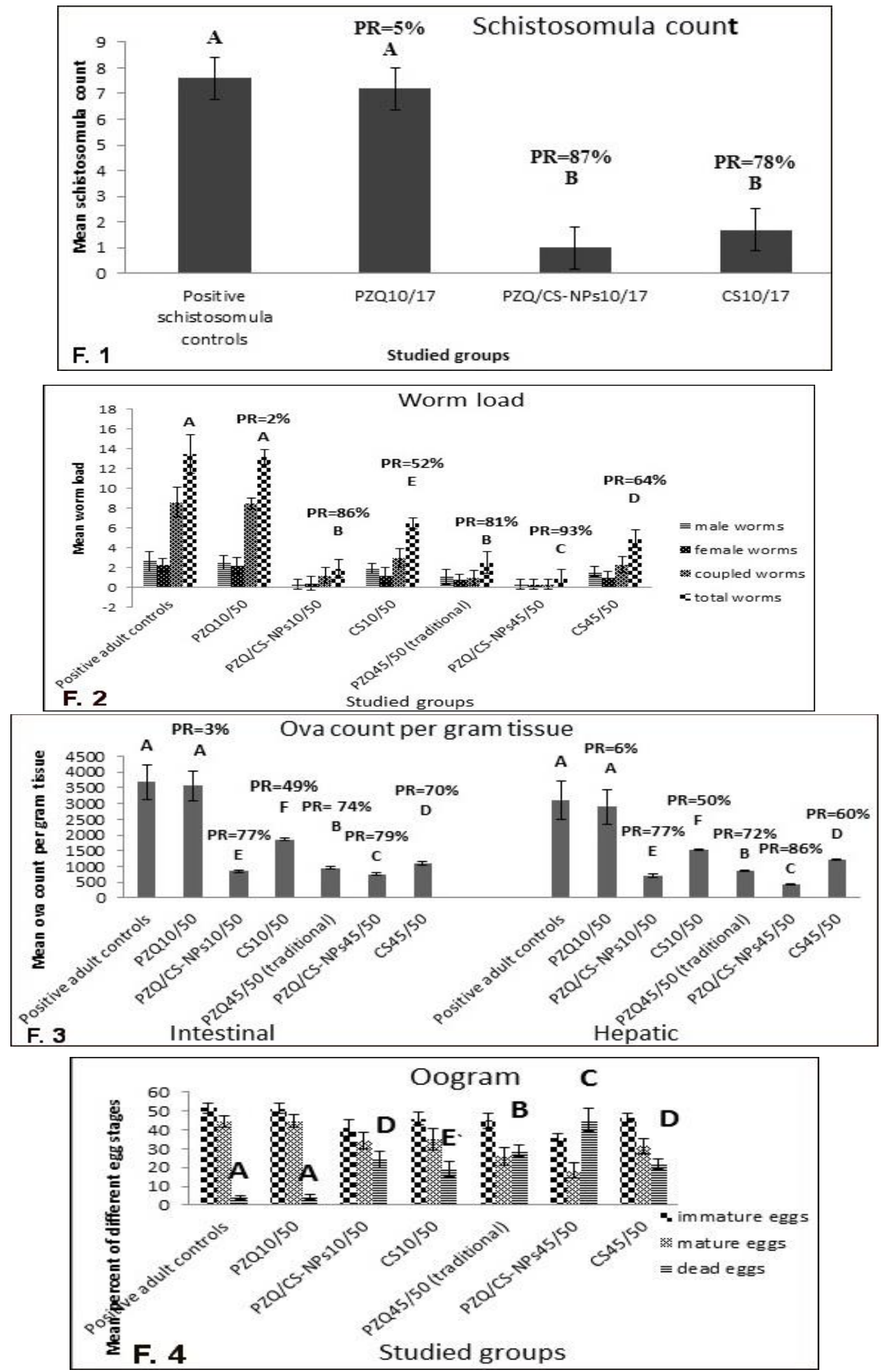

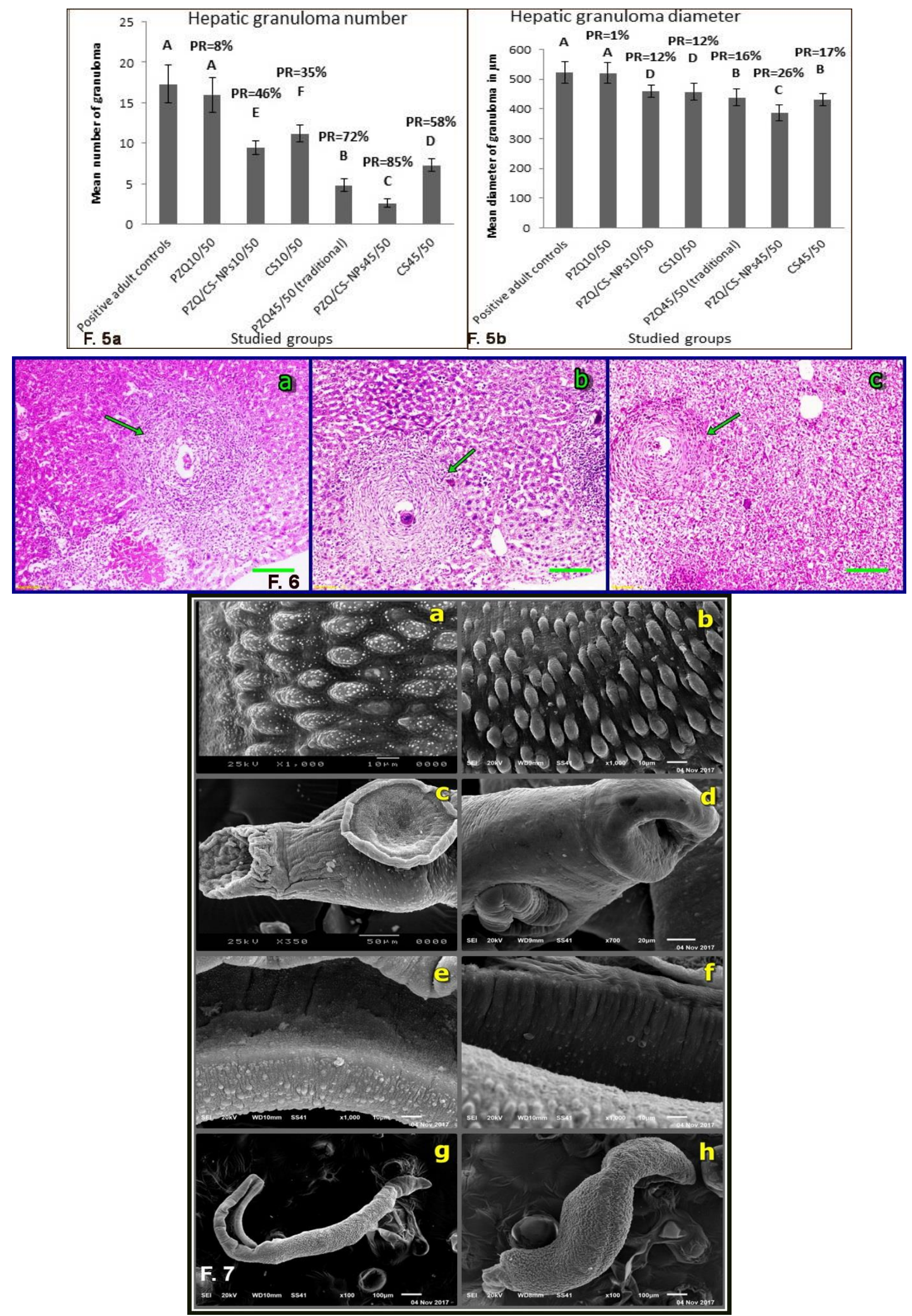